Bull. Mater. Sci., Vol. 3, Number 2, July 1981, pp. 235-245. (C) Printed in India.

\title{
Lattice imaging studies on the structure and non-random disorder in $\mathrm{SiC}$ crystals
}

\author{
S R SINGH and G SINGH \\ Department of Physics, Banaras Hindu University, Varanasi 221 005, India \\ MS received 5 November 1980
}

\begin{abstract}
A number of SiC crystals having extremely large period unit cells and onedimensionally disordered structures have been studied by $\mathrm{x}$-ray diffraction and lattice imaging in the electron microscope. It has been observed that structures of extremely large periodicities ( $>100 \mathrm{~nm}$ ) can be conveniently studied by lattice imaging technique which simply give continuous streaks in their x-ray diffraction patterns. This has been shown in the case of $150 \mathrm{R}, 150 \mathrm{R}_{\mathrm{A}}, 900 \mathrm{R}$ and another structure whose $c$-repeat period is more than $100 \mathrm{~nm}$. SiC crystals showing streaks along $c^{*}$-direction in $\mathrm{x}$-ray diffraction are found to possess either completely ordered structures with extremely large periodicities or non-random disorder.
\end{abstract}

Keywords. Polytypes; non-random disorder; intergrowth structures; lattice imaging.

\section{Introduction}

Structural studies of layer structures have generated a lot of interest because of the profusion of possible structural modifications in these materials and the role of ordering in crystallization of these structures. Ordering processes often lead to structures of large periodicities. Extremely large period structures pose formidable problems by the usual $\mathrm{x}$-ray crystallographic methods and require new techniques of investigation. Lattice imaging in the electron microscope has been found to be a very suitable and complimentary technique to $x$-ray diffraction for such studies. This paper deals with our recent work on the structure and one-dimensional disorder of a number of $\mathrm{SiC}$ structures.

Long periodicities in crystals could originate from more than one factor. In some cases, the basis is undoubtedly thermodynamic. The intermetallic systems such as $\mathrm{CuAu}$ show the ordering of atoms on several sublattices at regular intervals. The operative factor in such cases (Sato and Toth 1961, 1962; Sato et al 1967) is a lowering of the total electronic energy by decreasing the size of the Brillouin zone. In ionic compounds, ordering is due to the minimisation of the Coulombic component of the lattice energy.

The long periodicities $\sim 100 \mathrm{~nm}$ or more are found in the polytypes of non-metallic compounds such as $\mathrm{SiC}$ and $\mathrm{ZnS}$. The origin of such periodicities may be attributed to the growth kinetics rather than to an equilibrium state. These are binary tetra- 
hedral structures for which both f.c.c. and h.c.p. packing are possible. In the polytypes, the stacking of regular layers along $c$-axis changes at regular intervals from the cubic... ABCABC... sequence to the hexagonal.. ABAB... sequence. An isolated change in sequence or a set of uncorrelated changes, can be described in terms of stacking faults. If these changes in stacking sequence repeat at regular intervals, they generate a new translational unit of structure and define a new polytype. It is well-known from the extensive work on polytypism in $\mathrm{SiC}$ that such complex repeat sequences can be formed with great regularity. In the polytypes of these simple materials, there is no chemical difference between the layers in cubic and hexagonal stacking. Therefore, the change in stacking sequence arises only from the choice of sites with identical first-neighbour coordination.

Most of the polytypic substances possess planar faults giving rise to one-dimensional disorder or non-random disorder which are closely connected with their tendency to crystallise in different structures. Therefore, it was felt desirable to investigate the occurrence of extremely high periodicity and disorder in these substances and thereby try to understand the mechanism of ordering into perfect crystalline structures. This requires an experimental technique which could reveal local structural details at least at subunit-cell level. The x-ray diffraction which is by and large the most common technique for structural investigations, fails to reveal local structural fluctuations due to its averaging effect. Lattice imaging on the other hand has been found suitable for studying local fluctuations in structure and therefore the present investigation involves both the $x$-ray diffraction and lattice imaging techniques for studying structure and nature of disorder in SiC crystals. The first direct structure determination from high resolution electron microscope images was performed by Iijima and Allpress (1973).

\section{Experimental details}

High period structures of SiC are often found in syntactic coalescence with other polytypic structures and it becomes very difficult to get even minute pieces of the crystal which is exclusively composed of a particular high period structure. From such minute pieces of crystals used for $x$-ray examination, samples suitable for electron microscope examination were prepared by crushing them into powder form and dispersing the powder fragments on a holey carbon grid. No other sample preparation technique was possible with such minute crystals. These samples were examined in the Philips EM300 electron microscope equipped with a high resolution double tilt goniometer stage at an accelerating voltage of $100 \mathrm{kV}$. A thin crystal flake transparent to $100 \mathrm{kV}$ electrons was suitably oriented so that most of the $h O . l$ reflections are equally excited (zone-axis orientation). Bright-field lattice images were recorded by selecting in the objective aperture the direct beam along with symmetrical $00 . l$ reflections. The dark-field lattice images were obtained by beam deflection, without changing crystal orientation, so that objective aperture includes some basic $10 . l$ reflections together with a number of reflections of the high period structures.

\section{High periodicity and intergrowth structures}

During our systematic investigations on one-dimensional disorder in SiC crystals we have been selecting crystals showing continuous streaks along ho.l reciprocal lattice rows in X-ray diffraction and then examining them by lattice imaging technique. 
One of the crystals of this category gives $\mathrm{X}$-ray diffraction spots of some intergrown polytypes. A tiny crystal piece from parent crystal contains two polytypes in parallel intergrowth. Both the polytypes are rhombohedral with the same periodicity $(c=12.5 \mathrm{~nm})$ but in obverse and reverse orientation. This crystal piece consists predominantly of one of these structures (termed as $150 \mathrm{R}$ ) with only a small trace of the other in reverse orientation (to be termed subsequently as $150 R_{A}$ ) which is confined to only small part of the crystal under examination. The X-ray diffraction photographs taken from different fragments show slight variation in the relative intensities, of one structure relative to the other, indicating the varying proportions of $150 \mathrm{R}$ and $150 \mathrm{R}_{\mathrm{A}}$ in different fragments. Based on usual methods of structure determination of SiC polytypes, (Verma and Krishna 1966) a large number of probable structures were tried without success. The spots of $150 \mathrm{R}$ appear to split into many finer spots indicating the presence of super-periodicity (Ram et al 1973; Dubey et al 1973) based on $150 \mathrm{R}$. In view of these observations it was realised that the structure of $150 \mathrm{R}$ is of unusual type and lattice imaging studies may give useful information in its structure determination.

The most desirable condition (Cowley and Iijima 1972), for deriving any structural information, is to take bright-field lattice image by including symmetrical reflections in the objective aperture. Figure 1 shows a bright field lattice image formed by kinematically absent $O O . l$ reflections $(l \leqslant \pm 60)$ and the corresponding diffraction pattern depicting the objective aperture position is shown as inset. A closer look at figure 1 reveals the following: (a) The entire image consists of fringes of periodicity $1.25 \mathrm{~nm}$ out of which some are weak and others strong dark fringes. The smallest fringe spacing corresponds to $c / 3$ periodicity of $15 \mathrm{R}$ polytype and are due to conspicuously strong reflections at $15 \mathrm{R}$ spot positions. (b) The sequence of weak and strong dark fringes shows another periodicity of $12.5 \mathrm{~nm}$ which consists of ten $1.25 \mathrm{~nm}$ fringes. The $12.5 \mathrm{~nm}$ wide blocks correspond to consecutive reflections in $O O . I$ row and therefore represent $\mathrm{c} / 3$ periodicity of $150 \mathrm{R}$ polytype. (c) Every sixth block of $12.5 \mathrm{~nm}$ periodicity is faulted which is distinguished by a different sequence of $1.25 \mathrm{~nm}$ fringes in weak and strong dark contrast. These are marked $150 \mathrm{R}_{\mathrm{A}}$ in figure 1. The occurrence of these faulted blocks at regular intervals reveals a still higher periodicity of $75.5 \mathrm{~nm}$.

The X-ray diffraction intensities of $150 \mathrm{R}$ polytype shows that its structure is predominantly built up by stackings of $15 \mathrm{R}$ unit cells which is also confirmed from the lattice image where each $150 \mathrm{R}$ block contains ten $15 \mathrm{R}$ periodicities. This can bc so only when all the ten $15 \mathrm{R}$ unit cells are not identically stacked in $150 \mathrm{R}$ structure. This is indeed revealed in the lattice image where three of the ten $15 \mathrm{R}$ fringes are conspicuously in weak dark contrast compared to the rest. One distinct possibility of alternative stackings of $15 \mathrm{R}$ unit cells may be that some of them are in obverse orientation while others are in reverse orientation. The $15 \mathrm{R}$ unit cells in these two orientations may be represented by 23 and 32 in Zhdanov notation. Two consecutive unit cells of $15 R$ with 23 and 32 structures acquire nearly twinned orientation. Keeping this in view we realised that the sudden change in contrast in adjacent $15 R$ fringes in the lattice image may arise due to obverse and reverse orientations of $15 R$ unit cells and therefore correspond to 23 and 32 or vice-versa in the Zhdanov symbol of $150 \mathrm{R}$ structure. Treating $15 \mathrm{R}$ fringes in weak dark contrast as 32 and 23 and those in strong dark contrast as 23 and 32 respectively we arrive at the following two structures of $150 \mathrm{R}$ as read from bright field lattice image: 


$$
\begin{aligned}
& \text { (i) }\left[(23)_{3} 322332(23)_{3} 32\right]_{3} \text {, } \\
& \text { (ii) }\left[\begin{array}{llllll}
(32)_{3} & 23 & 32 & 23 & (32)_{3} & 23
\end{array}\right]_{3} \text {. }
\end{aligned}
$$

These two represent the same structure in obverse and reverse orientation and therefore are indistinguishable in X-ray diffraction. Therefore the intensities of $l O . l$ reflections were calculated for the above structure. The match between calculated and observed $x$-ray intensities is found to be excellent. Therefore, the above structure was found correct. The atomic positions of $\mathrm{Si}$ and $\mathrm{C}$ atoms can be derived from the Zhdanov symbol of the 150R structure.

Similarly, treating these fringes in the same way as in other $150 \mathrm{R}$ blocks, the structure corresponding to the faulted blocks represents an alternative $150 \mathrm{R}$ structure

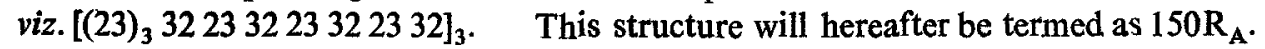
The calculated intensities of $150 \mathrm{R}_{\mathrm{A}}$ also match well with the intensities of all the observable $l O . l$ reflections of $150 \mathrm{R}_{\mathrm{A}}$. The $150 \mathrm{R}$ and $150 \mathrm{R}_{\mathrm{A}}$ structures are mutually in obverse and reverse orientation.

As mentioned earlier, the lattice image shows regular occurrence of one block of $150 \mathrm{R}_{\mathrm{A}}$ after every five blocks of $150 \mathrm{R}$, thereby giving rise to a superperiod of $75 \cdot 5 \mathrm{~nm}$. However, the superperiodicity inferred from the fine structure of $X$-ray spots corresponds roughly to the same periodicity as observed in the lattice image. Therefore it is reasonable to believe that few units of $75.5 \mathrm{~nm}$ periodicity are a part of high period structure.

Using established imaging code for $15 R$ fringes in weak and strong dark contrast which was validated by $X$-ray diffraction intensity observations of $150 \mathrm{R}$ and $150 \mathrm{R}_{\mathrm{A}}$ structure, one can reliably arrive at the following structure of the superperiod.

$$
\left[\left\{(23)_{3} 322332(23)_{3} 32\right\}_{5} \quad\left\{(23)_{3} 32233223322332\right\}\right]
$$

which is finally identified as $900 \mathrm{R}$ and the complete structure is described by repeating the above Zhdanov sequence three times. It may also be noted from figure 1 that one of the $12.5 \mathrm{~nm}$ blocks is faulted and has the structure (23) $32(23)_{3} 32$ which is different from those of the $150 \mathrm{R}$ and $150 \mathrm{R}_{\mathrm{A}}$ polytypes.

\section{Another extremely large period polytype of SiC}

Figure 2 shows the $10 . l$ row of $x$-ray diffraction pattern along with a dark-field lattice image of an extremely large period polytype. The corresponding electron diffraction pattern is superimposed on the image though not in proper orientation. The electron diffraction pattern from this crystal is as confusing as that of $\mathrm{x}$-ray diffraction so far as the determination of the periodicity is concerned. Both the techniques indicate one-dimensionally disordered structure. However, the lattice image clearly shows that the crystal is not disordered but has such a high periodicity (of about $124 \mathrm{~nm}$ ) that its diffraction spots merge to form streaks. The high period block itself consists of a sequence of fringes corresponding to $6 \mathrm{H}, 15 \mathrm{R}, 21 \mathrm{R}$ and $4 \mathrm{H}$ polytypes respectively.

On the basis of our observations on intergrowth structures of SiC, it may be said that crystals having coalesced polytype structures in parallel orientation contain superperiod structures in the transition region between coalesced structures which are 


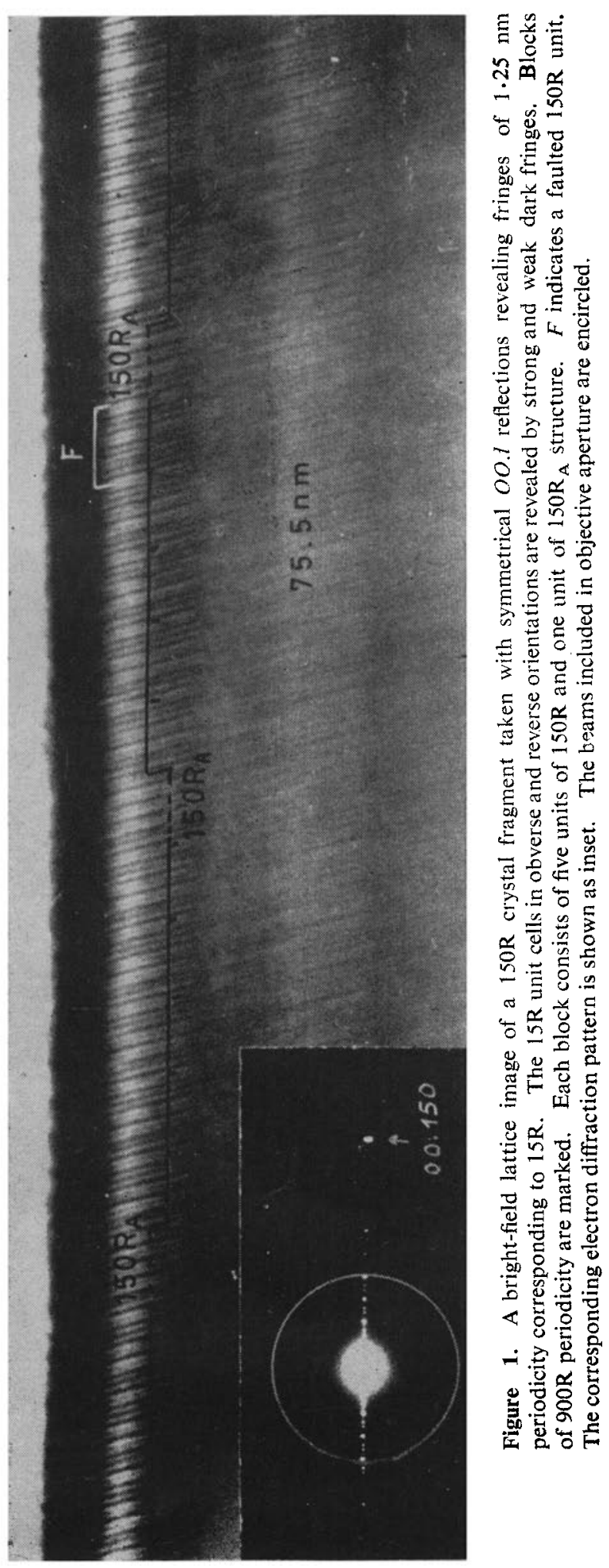




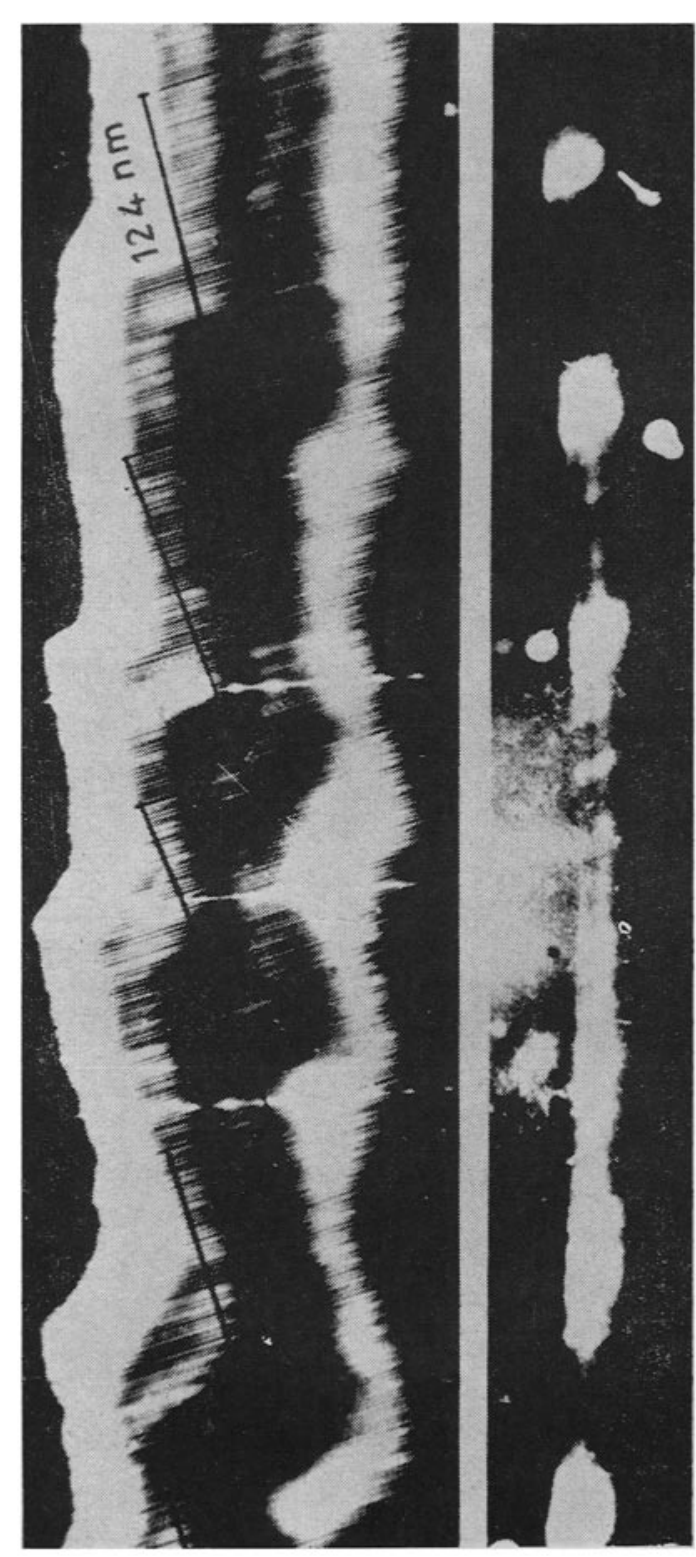

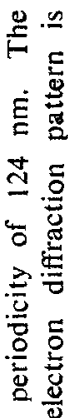

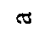

몰

点

范告

霑

焉高

离.

ㅎํㅇ

○

品

.

通

象

흠

흥 政 害守

飞驾

ن哭总

용

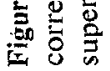


built up by unit cells of the adjoining structures. Therefore, the transition from one structure to another is not abrupt. Instead, there seems to be a boundary region of substantial extent where unit cells of the two adjoining structures stack in a definite fashion to create a large period structure. The transition region is of substantial thickness because it contain sufficient number of unit cells of the superperiod structure to give rise to characteristic X-ray diffraction pattern. In this context, Jepps et al (1979) have been able to analyse a 9R structure occurring in a very limited region in a $\beta$-SiC matrix in their phase transformation studies with the help of lattice imaging.

\section{Disorder}

The occurrence of continuous streaks along hO.l rows has been observed in diffraction studies of SiC crystals prepared in a number of different ways (Verma and Krishna 1966; Jagodzinski 1954; Shinozaki and Kinsman 1976; Sato and Shinozaki 1975 , etc.) and can be considered to arise either due to random disorder of layers or due to lack of resolution of $x$-ray reflections from extremely large period structures (Verma 1957). Lattice imaging plays an important role in the later case and reveals the long period structures very nicely (Dubey et al 1977; Singh and Singh 1980). Some crystals of first category are characterized by intensity modulations in continuous streaks which cannot be ascribed due to a particular periodicity. With the help of lattice imaging and x-ray diffraction, it has been found that the mode of disorder is non-random which is responsible for the observed intensity modulations of the streaks. We have studied a large number of such crystals, some of which are given below. Figure 3a shows the hO.l section of an electron diffraction pattern from one of the flakes of a crystal whose $\mathrm{x}$-ray diffraction pattern shows continuous streaks with intensity modulations. Figure $3 b$ shows a dark-field lattice image of the same crystal flake obtained from only $10 . l$ reflections. The following features can be noted from this image. (i) The image consists of line fringes most of which are of $15 \AA$ spacing corresponding to $c$-periodicity of the $6 \mathrm{H}$ polytype as was also expected on the basis of electron diffraction pattern. (ii) The sequence of $6 \mathrm{H}$ fringes are frequently interrupted by fringes of different spacings corresponding to c-periodicities of $15 \mathrm{R}, 21 \mathrm{R}$ and occasionally $4 \mathrm{H}$ polytype. (iii) The $15 \mathrm{R}$ fringes are clubbed together to form black bands. They occur as thin lamela (few unit cells) in parallel intergrowth with other polytype blocks and are conspicuous by their dark contrast. (iv) There are two distinct groups of $15 \mathrm{R}$ and $6 \mathrm{H}$ fringes (marked 1 and 3 in figure $3 \mathrm{~b}$ ) which occur more frequently with stacking sequence $(15 R)_{2}(6 \mathrm{H})_{1}(15 R)_{4}$ and $(15 \mathrm{R})_{1}(6 \mathrm{H})_{2}(15 \mathrm{R})_{2}$. Here subscripts indicate the number of spacings of corresponding polytypes. (v) The black bands occur in a quasi-periodic way at intervals of $35 \mathrm{~nm}$ (marked in figure $3 \mathrm{~b}$ ). This generates an average lattice of periodicity $\sim 35 \mathrm{~nm}$ and the discrete spots superposed on a continuous streak in figure $3 a$ correspond to this average lattice. In addition, there are other diffuse spots which may not be ordinarily correlated with periodicities observed in the lattice image. (vi) There are other groups of fringes of $6 \mathrm{H}$ and $15 \mathrm{R}$ spacings showing a favoured stacking sequence such as those marked 2 in figure $3 \mathrm{~b}$. They are not few but are far apart and therefore are not able to scatter in phase with the result that they fail to produce their characteristic diffraction spots. The above observations clearly indicate that though the crystal has many faults, they do not occur randomly. Inslead, there is a tendency of ordering which extends only up to a limited extent. Therefore the distribution of faults is non-random. 
Figure $4 \mathrm{a}$ is a $h O . l$ section of electron diffraction of a crystal foil from a parent crystal whose $\mathrm{x}$-ray diffraction shows a heavily disordered $6 \mathrm{H}$ structurc. The electron diffraction pattern contains closely spaced spots characteristic of intergrowth structures of different periodicities. Some of the reflections are sharp while others are diffuse and elongated which may be either due to distribution of planar faults in a complex manner (Cowley 1976) or due to a crystal having unit cells of a definite shape and size but the atomic distribution changes from one unit cell to another (Wilson 1942).

Figure $4 \mathrm{~b}$ is the dark-field lattice image of the crystal flake obtained from 10.1 reflections alone. Most of the fringes in this image correspond to $c$-periodicity of the 6H polytype but their stackings are frequently interrupted by fringes corresponding to other common polytypes. The unit cells of common polytypes $(6 \mathrm{H}, 15 \mathrm{R}, 21 \mathrm{R}$ etc.). show a tendency of ordering extending up to different extents. The tendency of a prominent black fringe to occur at regular intervals of $\sim 70 \mathrm{~nm}$ in the image is shown by marking the blocks defined by these fringes. On careful observation it is observed that the sequence of fringe spacings in individual blocks are not identical. However adjacent blocks show a tendency of having similar sequence of fringe spacings. It may be mentioned here that comparatively sharp reflections in the electron diffraction pattern (figure $4 \mathrm{a}$ ) correspond to $\sim 70 \mathrm{~nm}$ periodicity. This clearly indicates that periodic blocks observed in figure $4 \mathrm{~b}$ are represented in the diffraction pattern even though their contents are not strictly identical to each other. This observation is somewhat similar to the observations of Wilson (1942) in hexagonal cobalt which gives partly sharp and partly diffuse reflections because the unit cells are found to be of similar shape and size but the distribution of atoms differs from one unit cell to another throughout the crystal. Therefore the general pattern of disorder is that the strictly periodic blocks fail to acquire identical internal structures.

One thing which comes out very clearly from the lattice images of these crystals is that random occurrence of stacking faults in a polytype structure is seldom found to be responsible for continuous streaks in X-ray diffraction patterns.

The second important feature of the one-dimensional disorder in SiC crystals observed from these lattice images is that distinct structural entities occur at strictly regular intervals thereby defining a lattice periodicity. Apparently each of such periodic blocks are built up of sequences of the same number and type of unit cells of common polytypes which are not identical in their distribution in each periodic block.

Let us now consider the implications of these observations in the disordered SiC crystals to the tendency of polytype formation in this substance. Though there are very subtle differences in the thermodynamic stabilities of even the most common polytype structures $6 \mathrm{H}, 15 \mathrm{R}, 4 \mathrm{H} ; 6 \mathrm{H}$ seems to be the most stable phase in $\alpha$-SiC structures. All other polytypes are believed to be metastable structures. Even in disordered crystals the formation of preferred sequences of unit cells of common polytypes creating repeat units of higher period structures and their frequent occurrence corroborates this belief. The growth of coalesced structures in SiC crystals, where different structures of sufficient size intergrow in parallel orientation so that they are able to diffract $\mathrm{X}$-rays independently as individual crystals is a similar phenomenon taking place at the macroscopic level to what happens in the disordered $\mathrm{SiC}$ crystals at the unit cell level. The formation of polytypes with periodicities ranging say up to $10 \mathrm{~nm}$ or so may perhaps be conceived as metastable phases. How- 
$\widehat{\varrho}$

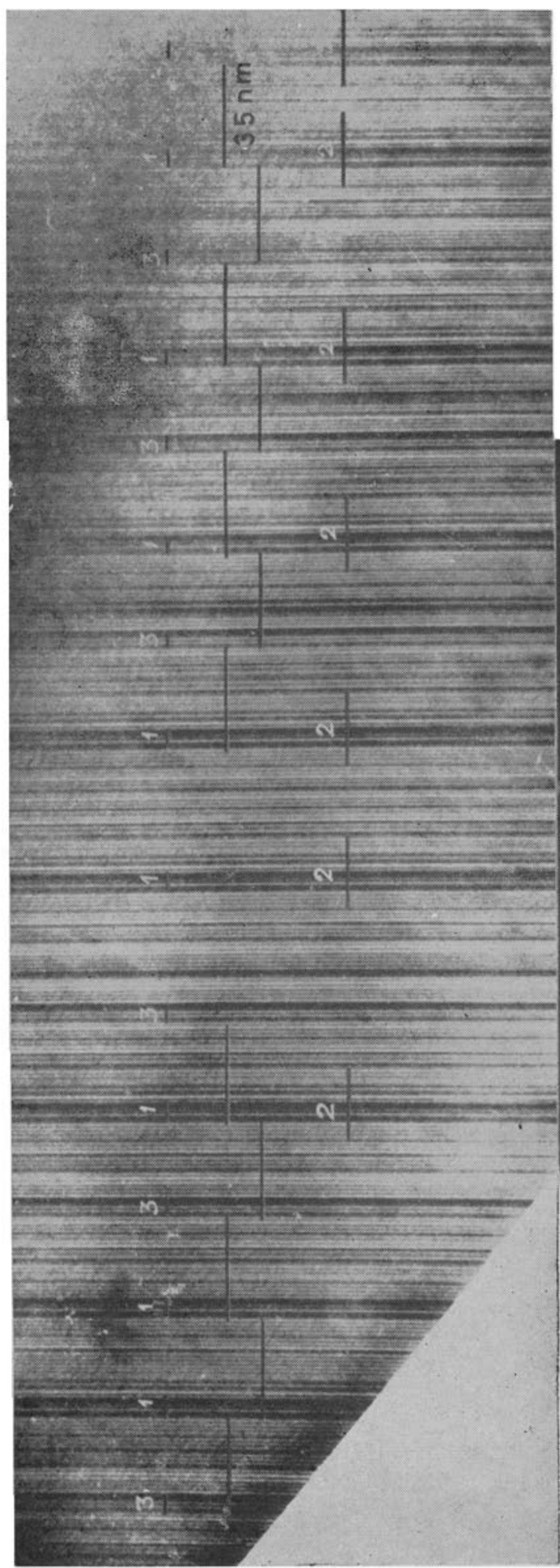

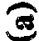

若密

品

棺冚

品

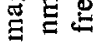

$=m$

.ّ

픔

更

궁

눈

宩

$<\frac{\pi}{3}$

金淃

$\dot{0}$

要歌

画

喝

IE

:

$\approx$ ठ ठ

ह

踏

몽

冚莺

퐁

F

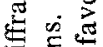

홍

5

要的

$\frac{}{4}$

42

들

记

ह

$\rightarrow$.

궁

는

F密

ส응

2 .

问柴

-

西

点电 
$\widehat{\mathrm{e}}$

๔

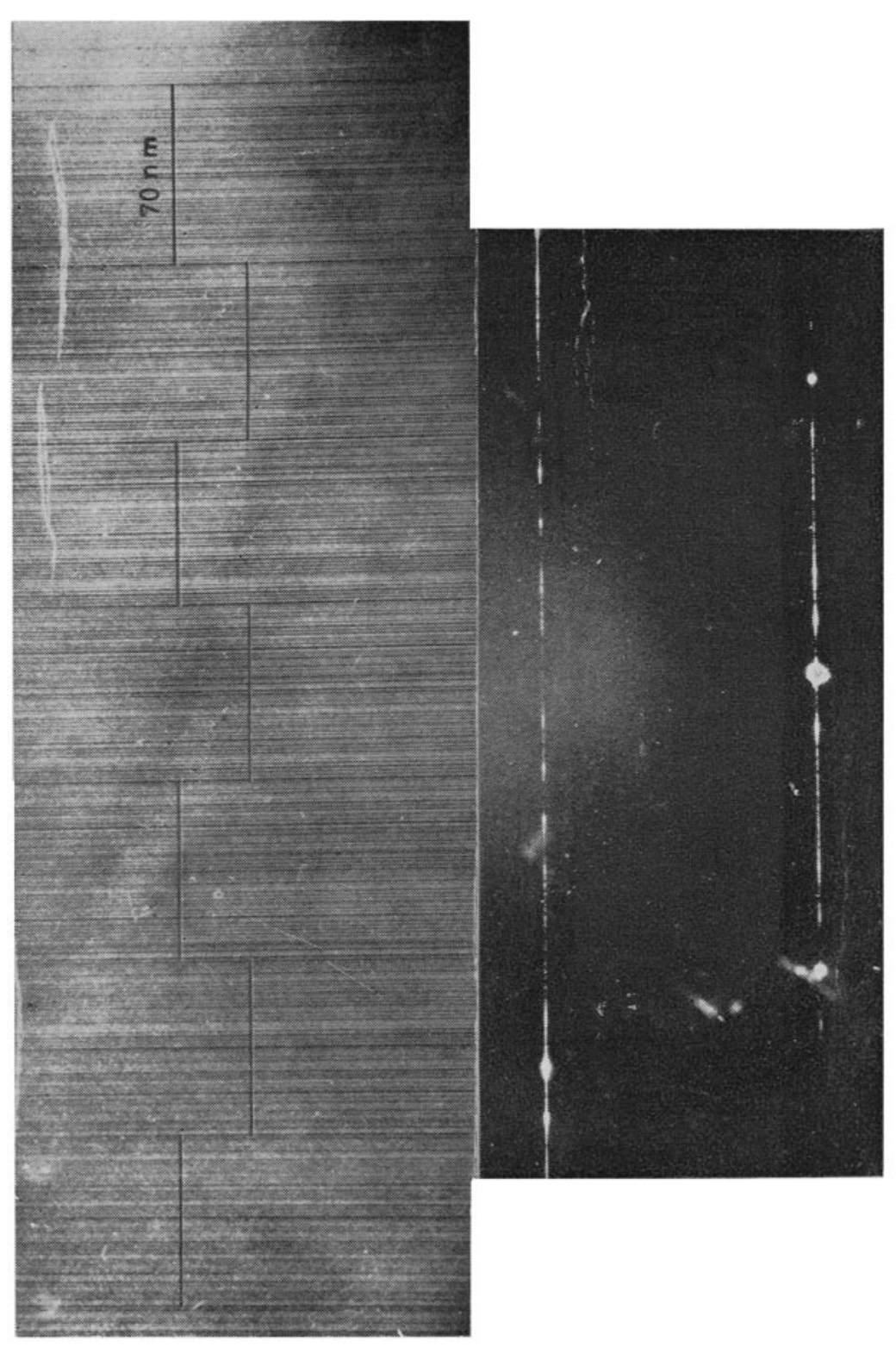

를 泀

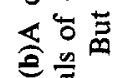

赔.

훙 焉

政

픈

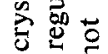

T

วั

过

훙ㅇㅇ응

음

․ㅡㄹ

पै

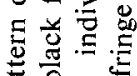

至.

ธิ

迅

穵 0 को

동 量

호을

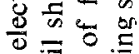

흥

휴 훙

氙氙总

$\rightarrow \&$ \&

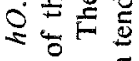

๘ิ

政

$+\Xi$ 至

造导

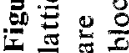


ever, the nature of forces responsible for the creation of periodicities of the order $100 \mathrm{~nm}$ or more which are so clearly manifested both in the lattice images and the $\mathrm{X}$-ray diffraction remains obscure.

\section{Conclusion}

Lattice imaging technique has been shown to play a very important role in investigating extremely large period structures which are difficult by $\mathrm{x}$-ray diffraction due to lack of resolution of closely spaced spots. It has also been found to play a complimentary role in determining crystal structures of high periodicities which would have been almost impossible by $x$-ray diffraction alone. The nature of disorder particularly one-dimensional disorder can be studied in greater detail by this technique.

\section{References}

Cowley J M 1976 Acta Crystallogr. A32 83, 88

Cowley J M and lijima S $1972 Z$. Naturforsch. A27 445

Dubey M, Ram U S and Singh G 1973 Acta Crystallogr. B29 1548

Dubey M, Singh G and Van Tendelloo G 1977 Acta Crystallogr. A33 276

Iijima S and Allpress J G 1974 Acta Crystallogr. A30 22

Jagodzinski H 1954 Acta Crystallogr. 7300

Jepps N W, Smith D J and Page T F 1979 Acta Crystallogr. A35 916

Ram U S, Dubey M and Singh G 1973 Z. Kristallogr. 137341

Sato H and Shinozaki S 1975 Mater. Res. Bull. 10257

Sato H and Toth R S 1961 Phys. Rev. 1241833

Sato H and Toth R S 1962 Phys. Rev. 127469

Sato H, Toth R S and Honjo G 1967 J. Phys. Chem. Solids 28137

Shinozaki S and Kinsman KR 1976 34th Annual Proceeding Electron Microscopy Society of America, Miami Beach (ed) G, W Bailey, p.652

Singh S R and Singh G 1980 Acta Crystallogr. A36 779

Verma A R 1957 Proc. R. Soc. London A240 462

Verma A R and Krishna P 1966 Polymorphism and polytypism in crystals (New York: Wiley)

Wilson A J C 1942 Proc. R. Soc. London A180 277 